\title{
Dead Sea drawdown and monsoonal impacts in the Levant during the last interglacial
}

\author{
Adi Torfstein ${ }^{\mathrm{a}, \mathrm{b}, \mathrm{c}, *}$, Steven L. Goldstein ${ }^{\mathrm{a}, \mathrm{d}}$, Yochanan Kushnir ${ }^{\mathrm{a}}$, Yehouda Enzel ${ }^{\mathrm{b}}$, \\ Gerald Haug ${ }^{\mathrm{e}}$, Mordechai Stein ${ }^{\mathrm{f}}$ \\ a Lamont-Doherty Earth Observatory of Columbia University, 61 Rt. 9W, Palisades, NY 10964, USA \\ ${ }^{\mathrm{b}}$ Institute of Earth Sciences, Hebrew University of Jerusalem, Jerusalem 91904, Israel \\ ${ }^{c}$ Interuniversity Institute of Marine Sciences, Eilat 88103, Israel \\ d Department of Earth and Environmental Sciences, Columbia University, 61 Rt. 9W, Palisades, NY 10964, USA \\ e ETH Zürich, Geologisches Institut, NO G 51.1, Sonneggstrasse 5, 8092 Zürich, Switzerland \\ ${ }^{\mathrm{f}}$ Geological Survey of Israel, 30 Malkhe Israel Street, Jerusalem 95501, Israel
}

\section{A R T I C L E I N F O}

\section{Article history:}

Received 7 August 2014

Received in revised form 29 November 2014

Accepted 4 December 2014

Available online 9 January 2015

Editor: J. Lynch-Stieglitz

\section{Keywords:}

last interglacial

paleoclimate

Dead Sea

sapropel

Levant

African monsoon

\begin{abstract}
A B S T R A C T
Sediment cores recovered by the Dead Sea Deep Drilling Project (DSDDP) from the deepest basin of the hypersaline, terminal Dead Sea (lake floor at $\sim 725 \mathrm{~m}$ below mean sea level) reveal the detailed climate history of the lake's watershed during the last interglacial period (Marine Isotope Stage 5; MIS5). The results document both a more intense aridity during MIS5 than during the Holocene, and the moderating impacts derived from the intense MIS5e African Monsoon. Early MIS5e ( $133-128 \mathrm{ka})$ was dominated by hyperarid conditions in the Eastern Mediterranean-Levant, indicated by thick halite deposition triggered by a lake-level drop. Halite deposition was interrupted however, during the MIS5e peak ( $\sim 128-122 \mathrm{ka}$ ) by sequences of flood deposits, which are coeval with the timing of the intense precession-forced African monsoon that generated Mediterranean sapropel S5. A subsequent weakening of this humidity source triggered extreme aridity in the Dead Sea watershed and resulting in the biggest known lake level drawdown in its history, reflected by the deposition of thick salt layers, and a capping pebble layer corresponding to a hiatus at $\sim 116-110 \mathrm{ka}$. The DSDDP core provides the first evidence for a direct association of the African monsoon with mid subtropical latitude climate systems effecting the Dead Sea watershed. Combined with coeval deposition of Arabia and southern Negev speleothems, Arava travertines, and calcification of Red Sea corals, the evidence points to a climatically wet corridor that could have facilitated homo sapiens migration "out of Africa" during the MIS5e peak. The hyperaridity documented during MIS5e may provide an important analogue for future warming of arid regions of the Eastern Mediterranean-Levant.
\end{abstract}

(C) 2014 Elsevier B.V. All rights reserved.

\section{Introduction}

The Dead Sea is a hypersaline, terminal lake occupying the lowest surface on Earth's continents, whose water surface is currently at $\sim 428 \mathrm{~m}$ below mean sea level (mbsl), and whose large $\left(\sim 40000 \mathrm{~km}^{2}\right)$ watershed spans the Mediterranean and the Saharo-Arabian climate zones (Fig. 1). Its volume increases during glacials and declines during interglacials, with amplified positive and negative responses of its levels, respectively. The mineralogy, grain sizes, and chemical and isotope compositions of the lake deposits reflect the regional climate during past glacial-interglacial

\footnotetext{
* Corresponding author at: Institute of Earth Sciences, Hebrew University of Jerusalem, Jerusalem 91904, Israel.

E-mail address: adi.torf@mail.huji.ac.il (A. Torfstein).
}

cycles (Enzel et al., 2008; Stein, 2001). Studies of the Holocene Dead Sea, the last glacial Lake Lisan, and earlier Pleistocene lake cycles (i.e., Lake Amora), based mainly on exposed deposits along the lake margins, show close connections between Northern Hemisphere $(\mathrm{NH})$ climate changes and water levels, water chemistry, and sediment lithology on glacial-interglacial to millennial time-scales (Bartov et al., 2003; Haase-Schramm et al., 2004; Kushnir and Stein, 2010; Prasad et al., 2004; Torfstein et al., 2013b). For example, since the Last Glacial Maximum and during the last deglaciation ( $24-11 \mathrm{ka})$, lake level dropped from its glacial high-stand of $\sim 160 \mathrm{mbsl}$ to below $460 \mathrm{mbsl}$ at the BøllingAllerød (Stein et al., 2010), stabilizing at a Holocene (average) level of $\sim 400 \mathrm{mbsl}$. However, margin exposures cover limited timeintervals and mainly sediments deposited during the last glacial Lake Lisan (e.g., Bartov et al., 2002); continuous sedimentation 

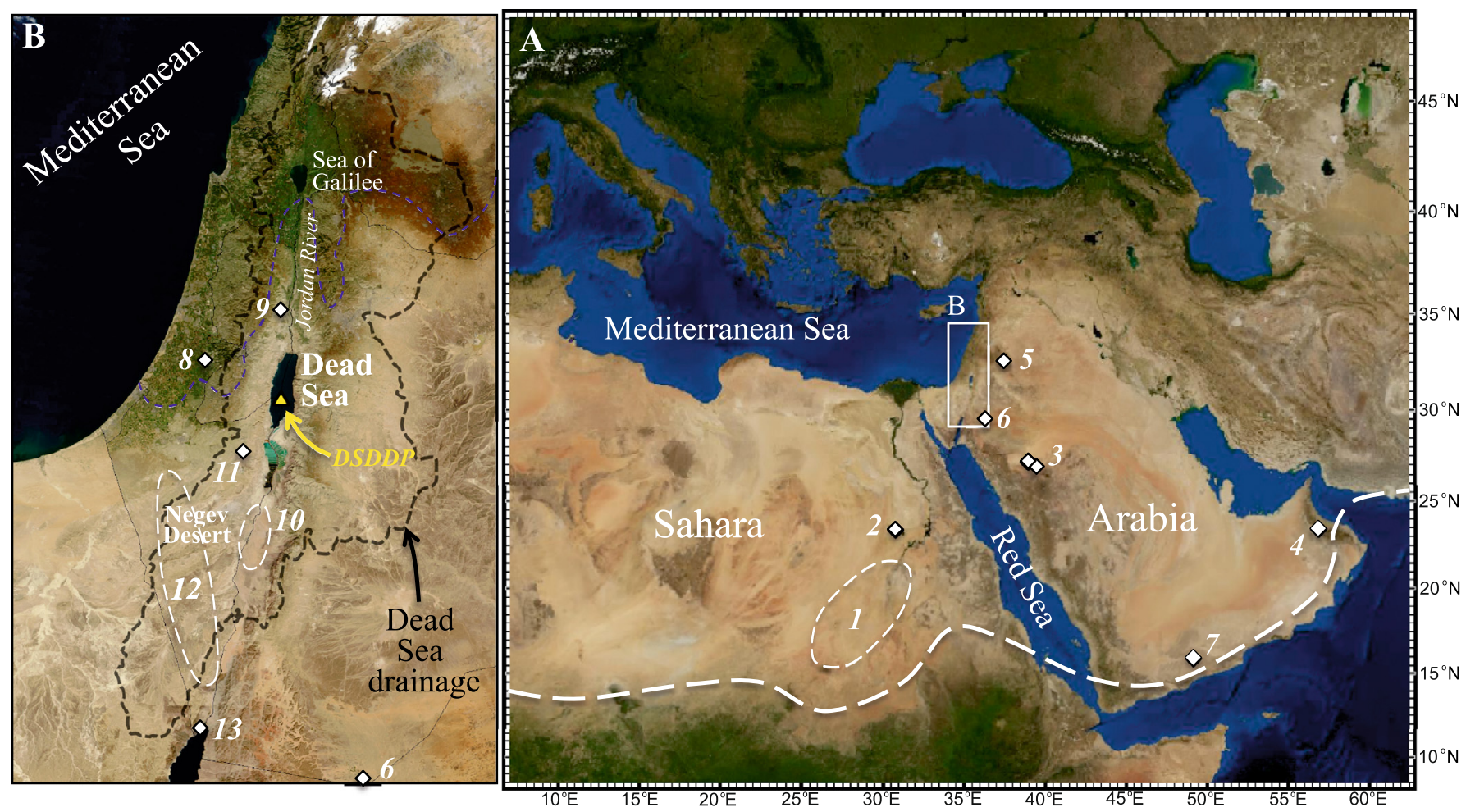

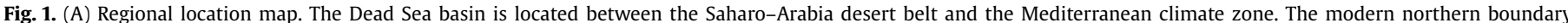

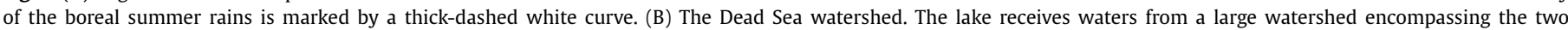

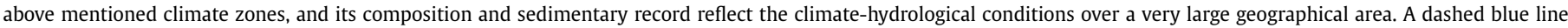

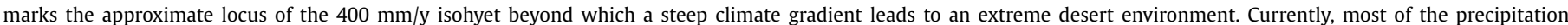

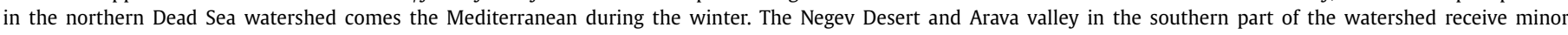

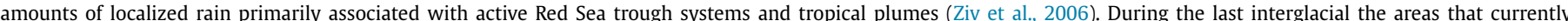

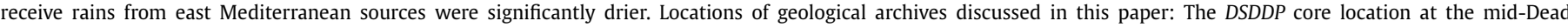

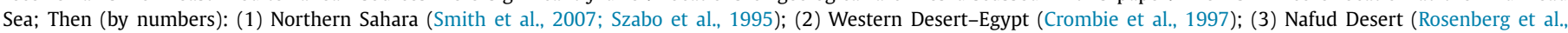

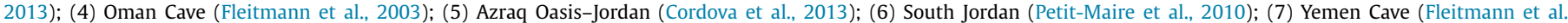

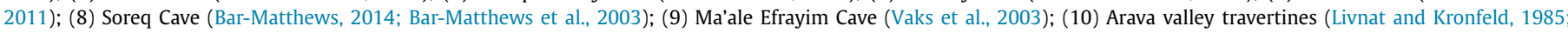

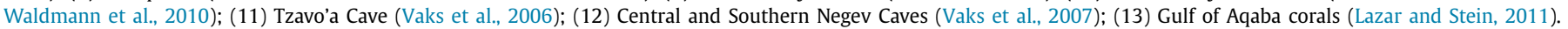

has occurred only in the deepest part of the lake. The Dead Sea Deep Drilling Project (DSDDP) conducted in 2010-2011 under the auspices of the Intercontinental Scientific Drilling Program (ICDP), recovered a detailed sedimentary record going back to MIS7, from the deepest basin of the modern lake (Neugebauer et al., 2014). The drilling took place at a lake depth of $297 \mathrm{~m}$, and the coring reached $456 \mathrm{~m}$ below the lake floor (mblf), recovering otherwise hidden sedimentary sections of the last interglacial low-stand lake.

This study presents a $200 \mathrm{kyr}$ climate record from the DSDDP core and focuses on the climate history of the Levant during the last interglacial MIS5. This time interval has relevance in the context of climate models indicating a more arid Middle East with increasing global temperatures (e.g., Held and Soden, 2006), thus implying increased future fresh water scarcity in a water-starved and politically unstable region. Because the last interglacial peak (MIS5e, between $\sim 135-116 \mathrm{ka}$; Lisiecki and Raymo, 2005) was characterized by stronger insolation, warmer global temperatures, higher sea levels, and smaller continental ice sheets compared to the Holocene, the DSDDP core record provides a test of such predictions; thus, it serves as an analogue for a future warmer world. Our results show both extreme hyperaridity during MIS5e, including an unprecedented drawdown of Dead Sea water levels, as well as important impacts of a particularly strong precessioncontrolled African monsoon that generated a major sapropel (S5; Rohling et al., 2002; Rossignol-Strick, 1985) in the eastern Mediterranean.

\section{Sedimentary records of the Dead Sea lakes and their hydroclimate connection}

Previous studies on marginal terraces and short cores drilled along the Dead Sea shores have revealed that the lithology of the lacustrine sections directly reflects the watershed hydroclimatology (e.g., Begin et al., 1974; Bookman (Ken-Tor) et al., 2004; Haase-Schramm et al., 2004; Migowski et al., 2006; Stein et al., 1997; Yechieli et al., 1993). A wet hydrological regime is represented by summer deposition of primary (evaporitic) aragonitic laminae. These laminae alternate with detrital quartz and calcite silt grains from desert dust origin (Haliva-Cohen et al., 2012), that accumulated in the Dead Sea watershed and was washed into the lake during winters. The alternating aragonite and detritus couplets comprising the aad facies (Figs. 2, S1) reflect annual cycles of deposition (Begin et al., 1974; Prasad et al., 2004). The Ca-chloride Dead Sea brine is poor in bi-carbonate and sulfate, required for aragonite or gypsum precipitation. These ions are supplied by freshwaters entering the lake during wet periods in the watershed, and support the precipitation of aragonite and gypsum, which are characteristic of wet glacial intervals in the Dead Sea lacustrine deposits (Stein et al., 1997; Torfstein et al., 2008, 2005). During arid periods, smaller amounts of bicarbonate and sulfate are supplied to the lake and the deposition is dominated the silty quartz and calcite grains comprising the laminated detritus (the ld facies; Haliva-Cohen et al., 2012). This sediment comprises significant portions of the interglacial sequences exposed along the margins of the modern lake (e.g., 


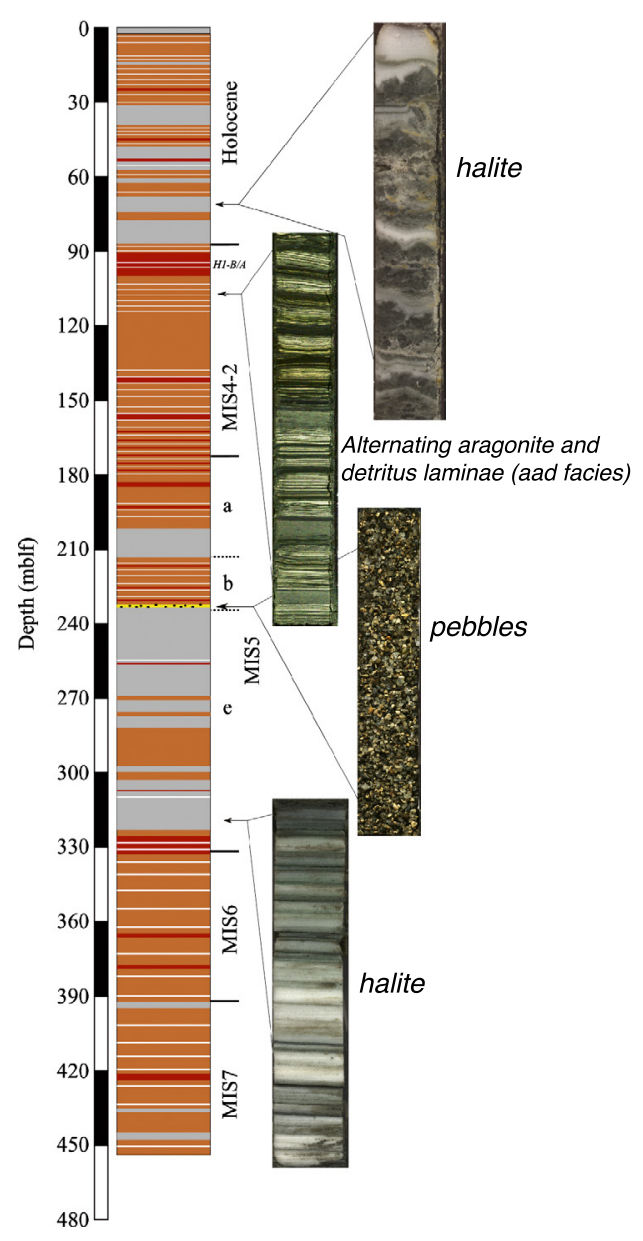

Fig. 2. Compiled columnar section and primary sedimentary facies. The main types of sediments comprising the DSDDP core are: (1) Alternating aragonite and detritus laminae (aad facies; brown-white lamination), characterizing periods of enhanced freshwater input; (2) Laminated silts (ld facies; brown), characterizing floods occurring during periods of relative aridity; (3) Halite (gray) and gypsum layers (red) marking lake level drops. A pebble unit ca. 235 mblf (yellow) marks the catastrophic low point of water elevations during MIS5. (For interpretation of the references to color in this figure legend, the reader is referred to the web version of this article.)

the Amora/Samra and early Holocene Formations; Migowski et al., 2006; Torfstein et al., 2009; Waldmann et al., 2009). Increasing aridity in the watershed is reflected by the deposition of gypsum, and hyperaridity along with significant lake level drops is reflected by deposition of halite. Thick sequences of halite have been recovered during shallow drilling along the Dead Sea margins to the base of the Holocene (Neev and Emery, 1967; Stein et al., 2010; Yechieli et al., 1993).

\section{U-Th ages and age model}

U-Th ages were obtained on primary aragonites that comprise thin laminae of the aad facies throughout the core. Analytical procedures are summarized in the Supplementary Material. The approach to achieve U-Th (calendar) ages from the primary aragonites of the late Quaternary Dead Sea lacustrine sections was developed over several decades of research (Haase-Schramm et al., 2004; Kaufman, 1971; Kaufman et al., 1992; Schramm et al., 2000; Torfstein et al., 2013a). The Dead Sea aragonites have been called "dirty carbonates" because they contain some detrital Th and $\mathrm{U}$, and hydrogenous $\mathrm{Th}$, all requiring the appropriate corrections to the simple age equation. Here, we estimated the $\mathrm{U}$ and Th concentrations and isotope compositions of the Detritus End Member (DEM) for each individual set of coeval sam-

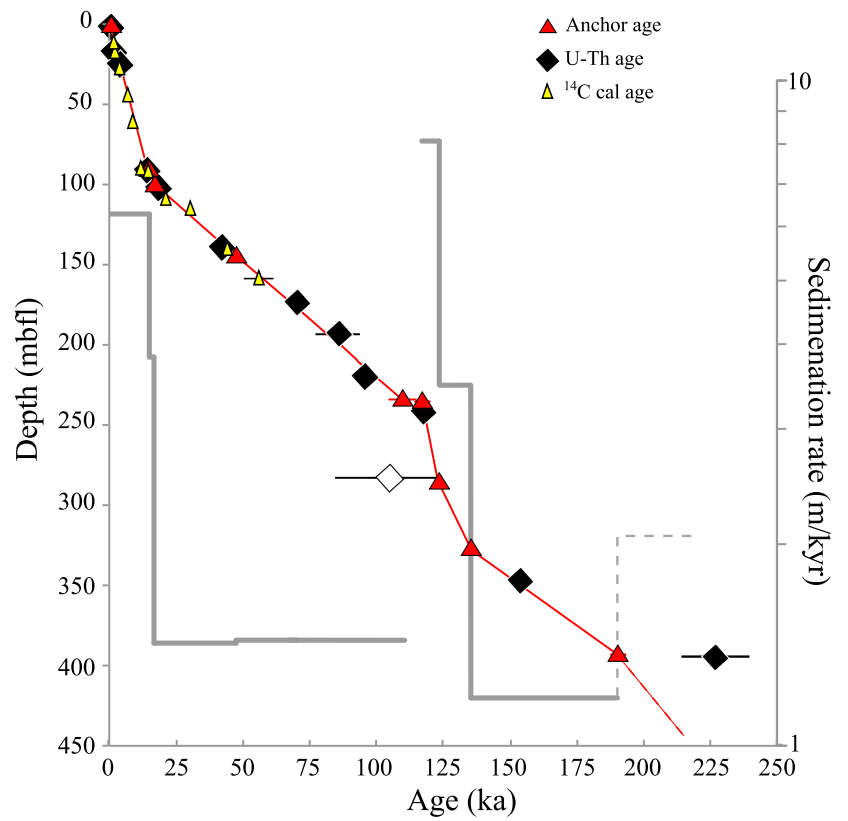

Fig. 3. Age model and sedimentation rates. Anchor ages (red triangles) were determined based on the compilation of U-Th ages, correlation of defined stratigraphic layers to well dated counterparts on the basins margins, and a fit between the oxygen isotope stratigraphy and main lithological facies to the LR04 (Lisiecki and Raymo, 2005) and Soreq Cave (Bar-Matthews et al., 2003; Grant et al., 2012) records (details in Supplementary Material). The sedimentation rates show large variations, whereby glacial and interglacial stages are characterized by low and high rates, respectively. Radiocarbon ages of organic material in the DSDDP core (yellow triangles) are from Neugebauer et al. (2014). (For interpretation of the references to color in this figure legend, the reader is referred to the web version of this article.)

ples. This was based on the intercept between the extrapolated $\left({ }^{234} \mathrm{U} /{ }^{238} \mathrm{U}\right)-\left({ }^{230} \mathrm{Th} /{ }^{238} \mathrm{U}\right)-\left({ }^{232} \mathrm{Th} /{ }^{238} \mathrm{U}\right)$ ratios of the samples and the secular equilibrium curve, under the premise that ${ }^{238} \mathrm{U},{ }^{234} \mathrm{U}$ and ${ }^{230} \mathrm{Th}$ are in secular equilibrium in detritus material (details in Torfstein et al., 2013a). The extracted DEM value is subsequently used to correct the individual sample ages (i.e., 'single sample ages'), with an assumed (conservative) uncertainty of 30\%, according to procedures described in Haase-Schramm et al. (2004) and Torfstein et al. (2013a). Because the impact of a potential hydrogenous Th component is limited to several hundreds of years and is negligible in samples older than several tens of thousands of years, we do not consider this component in the calculations. The measured ratios, and the calculated ages are listed in Table 1 .

The age-depth model for the DSDDP core (Fig. 3; Table 2) is based on the integration of the U-Th ages with oxygen isotope ratios in aragonite from the core, and lithological changes in the core, all of which are tied to key points in the LR04 and Soreq Cave $\delta^{18} \mathrm{O}$ records (Bar-Matthews et al., 2003; Grant et al., 2012; Lisiecki and Raymo, 2005), as well as correlations between distinct stratigraphic units to well-dated counterparts on the exposed margins of the Dead Sea basin (details in Supplementary Material).

\section{Chronology and stratigraphy of the DSDDP MIS5 interval}

From the DSDDP core litho-stratigraphy, a first order observation is that glacial terminations and interglacials are characterized by halite, gypsum, and laminated silt clastics (Fig. 2). The glacial intervals display thick sequences of the aad facies with some gypsum and silts during drier intervals (Fig. 2). Here, we focus on the last interglacial MIS5 interval (328-177 mblf), comprising thick halite sequences interspersed with intermittent gypsum, sequences of silt layers, and some sequences of the aad facies. As already noted, these lithologies document major climatic shifts ranging 
Table 1

U-Th data. Ages used for age model construction are highlighted in bold font. Rounded brackets mark activity ratios.

\begin{tabular}{|c|c|c|c|c|c|c|c|c|c|c|c|c|c|c|c|c|c|c|}
\hline $\begin{array}{l}\text { Sample } \\
\text { name }\end{array}$ & $\begin{array}{l}\text { Depth } \\
(\mathrm{m})\end{array}$ & $\begin{array}{l}\left({ }^{230} \mathrm{Th} /\right. \\
\left.{ }^{238} \mathrm{U}\right)\end{array}$ & $2 \sigma$ & $\begin{array}{l}\left({ }^{234} \mathrm{U} /\right. \\
\left.{ }^{238} \mathrm{U}\right)\end{array}$ & $2 \sigma$ & $\begin{array}{l}\left({ }^{230} \mathrm{Th} /\right. \\
\left.{ }^{232} \mathrm{Th}\right)\end{array}$ & $2 \sigma$ & $\begin{array}{l}\mathrm{U} \\
(\mathrm{ppm})\end{array}$ & $\begin{array}{l}\text { Th } \\
(\mathrm{ppm})\end{array}$ & $\begin{array}{l}{ }^{232} \mathrm{Th} / 238 \mathrm{U} \\
\text { (atomic) } \\
\text { detritus } \\
\text { correction }\end{array}$ & $\begin{array}{l}\text { Single } \\
\text { sample age } \\
\text { (y) }\end{array}$ & $2 \sigma(+)$ & $2 \sigma(-)$ & $\begin{array}{l}\text { Isochron } \\
\text { age } \\
\text { (y) }\end{array}$ & $2 \sigma(+)$ & $2 \sigma(-)$ & $\begin{array}{l}\text { Average } \\
\text { single } \\
\text { sample age } \\
\text { (y) }\end{array}$ & $\begin{array}{l}2 \sigma \\
\text { stderr }\end{array}$ \\
\hline $\begin{array}{l}\text { AT-105-d } \\
\text { AT-105-e }\end{array}$ & $\begin{array}{l}2.76 \\
2.76\end{array}$ & $\begin{array}{l}0.190 \\
0.278\end{array}$ & $\begin{array}{l}4.1 \mathrm{E}-04 \\
7.1 \mathrm{E}-04\end{array}$ & $\begin{array}{l}1.420 \\
1.379\end{array}$ & $\begin{array}{l}7 \mathrm{E}-04 \\
8 \mathrm{E}-04\end{array}$ & $\begin{array}{l}2.12 \\
2.13\end{array}$ & $\begin{array}{l}4 \mathrm{E}-03 \\
5 \mathrm{E}-03\end{array}$ & $\begin{array}{l}1.30 \\
1.75\end{array}$ & $\begin{array}{l}0.355 \\
0.701\end{array}$ & $\begin{array}{l}1.539 \\
1.539\end{array}$ & $\begin{array}{r}673 \\
1139\end{array}$ & $\begin{array}{r}80 \\
221\end{array}$ & $\begin{array}{r}73 \\
187\end{array}$ & -64 & 150 & -66 & 906 & 466 \\
\hline $\begin{array}{l}\text { AT-47-d } \\
\text { AT-47-c } \\
\text { AT-47-b }\end{array}$ & $\begin{array}{l}16.94 \\
16.94 \\
16.94\end{array}$ & $\begin{array}{l}0.415 \\
0.240 \\
0.173\end{array}$ & $\begin{array}{l}9.3 \mathrm{E}-04 \\
5.6 \mathrm{E}-04 \\
4.0 \mathrm{E}-04\end{array}$ & $\begin{array}{l}1.341 \\
1.421 \\
1.429\end{array}$ & $\begin{array}{l}1 \mathrm{E}-03 \\
8 \mathrm{E}-04 \\
1 \mathrm{E}-03\end{array}$ & $\begin{array}{l}2.77 \\
2.52 \\
2.98\end{array}$ & $\begin{array}{l}5 E-03 \\
6 E-03 \\
7 E-03\end{array}$ & $\begin{array}{l}2.13 \\
1.61 \\
0.73\end{array}$ & $\begin{array}{l}0.970 \\
0.467 \\
0.130\end{array}$ & $\begin{array}{l}1.381 \\
1.381 \\
1.381\end{array}$ & $\begin{array}{l}8478 \\
2167 \\
3534\end{array}$ & $\begin{array}{r}2426 \\
306 \\
268\end{array}$ & $\begin{array}{r}1867 \\
269 \\
249\end{array}$ & 1858 & 1909 & 1769 & 4727 & 3834 \\
\hline $\begin{array}{l}\text { AT-1 } \\
\text { AT-62-a }\end{array}$ & $\begin{array}{l}21.25 \\
21.25\end{array}$ & $\begin{array}{l}0.248 \\
0.832\end{array}$ & $\begin{array}{l}4.5 \mathrm{E}-04 \\
9.0 \mathrm{E}-04\end{array}$ & $\begin{array}{l}1.406 \\
1.376\end{array}$ & $\begin{array}{l}1 E-03 \\
5 E-04\end{array}$ & $\begin{array}{l}2.40 \\
8.18\end{array}$ & $\begin{array}{l}4 \mathrm{E}-03 \\
7 \mathrm{E}-03\end{array}$ & $\begin{array}{l}2.37 \\
1.75\end{array}$ & $\begin{array}{l}0.746 \\
0.543\end{array}$ & $\begin{array}{l}1.447 \\
1.447\end{array}$ & $\begin{array}{r}2266 \\
78297\end{array}$ & $\begin{array}{r}341 \\
17385\end{array}$ & $\begin{array}{r}296 \\
13172\end{array}$ & & & & & \\
\hline $\begin{array}{l}\text { AT-21-b } \\
\text { AT-21-d } \\
\text { AT-21-e } \\
\text { AT-21-f }\end{array}$ & $\begin{array}{l}92.20 \\
92.20 \\
92.20 \\
92.20\end{array}$ & $\begin{array}{l}0.225 \\
0.249 \\
0.239 \\
0.244\end{array}$ & $\begin{array}{l}4.1 \mathrm{E}-04 \\
6.0 \mathrm{E}-04 \\
3.7 \mathrm{E}-04 \\
3.8 \mathrm{E}-04\end{array}$ & $\begin{array}{l}1.467 \\
1.467 \\
1.458 \\
1.461\end{array}$ & $\begin{array}{l}1 E-03 \\
9 E-04 \\
9 E-04 \\
1 E-03\end{array}$ & $\begin{array}{l}7.00 \\
5.78 \\
6.41 \\
5.44\end{array}$ & $\begin{array}{l}1 \mathrm{E}-02 \\
1 \mathrm{E}-02 \\
9 \mathrm{E}-03 \\
8 \mathrm{E}-03\end{array}$ & $\begin{array}{l}1.97 \\
2.68 \\
1.91 \\
2.28\end{array}$ & $\begin{array}{l}0.194 \\
0.352 \\
0.217 \\
0.312\end{array}$ & $\begin{array}{l}1.447 \\
1.447 \\
1.447 \\
1.447\end{array}$ & $\begin{array}{l}12813 \\
13036 \\
13212 \\
12372\end{array}$ & $\begin{array}{l}491 \\
685 \\
589 \\
682\end{array}$ & $\begin{array}{l}473 \\
647 \\
562 \\
643\end{array}$ & 14067 & 1146 & 1135 & 12858 & 363 \\
\hline $\begin{array}{l}\text { AT-28-b } \\
\text { AT-28-c } \\
\text { AT-28-d }\end{array}$ & $\begin{array}{l}102.10 \\
102.10 \\
102.10\end{array}$ & $\begin{array}{l}0.318 \\
0.266 \\
0.281\end{array}$ & $\begin{array}{l}5.1 \mathrm{E}-04 \\
4.4 \mathrm{E}-04 \\
4.8 \mathrm{E}-04\end{array}$ & $\begin{array}{l}1.407 \\
1.437 \\
1.427\end{array}$ & $\begin{array}{l}1 \mathrm{E}-03 \\
9 \mathrm{E}-04 \\
8 \mathrm{E}-04\end{array}$ & $\begin{array}{l}3.86 \\
7.24 \\
5.67\end{array}$ & $\begin{array}{l}6 E-03 \\
1 E-02 \\
9 E-03\end{array}$ & $\begin{array}{l}2.95 \\
1.82 \\
2.16\end{array}$ & $\begin{array}{l}0.741 \\
0.204 \\
0.327\end{array}$ & $\begin{array}{l}2.171 \\
2.171 \\
2.171\end{array}$ & $\begin{array}{l}18149 \\
18119 \\
18196\end{array}$ & $\begin{array}{r}1295 \\
527 \\
711\end{array}$ & $\begin{array}{r}1198 \\
510 \\
682\end{array}$ & 18140 & 83 & 83 & 18155 & 45 \\
\hline $\begin{array}{l}\text { AT-42-a } \\
\text { AT-42-c } \\
\text { AT-42-e } \\
\text { AT-42-c-dup }\end{array}$ & $\begin{array}{l}139.27 \\
139.27 \\
139.27 \\
139.27\end{array}$ & $\begin{array}{l}0.507 \\
0.508 \\
0.539 \\
0.513\end{array}$ & $\begin{array}{l}7.1 \mathrm{E}-04 \\
6.4 \mathrm{E}-04 \\
1.4 \mathrm{E}-03 \\
1.0 \mathrm{E}-03\end{array}$ & $\begin{array}{l}1.444 \\
1.449 \\
1.402 \\
1.448\end{array}$ & $\begin{array}{l}1 E-03 \\
1 E-03 \\
1 E-03 \\
1 E-03\end{array}$ & $\begin{array}{c}13.2 \\
14.4 \\
7.00 \\
14.9\end{array}$ & $\begin{array}{l}2 \mathrm{E}-02 \\
2 \mathrm{E}-02 \\
2 \mathrm{E}-02 \\
2 \mathrm{E}-02\end{array}$ & $\begin{array}{l}1.68 \\
1.66 \\
2.07 \\
1.65\end{array}$ & $\begin{array}{l}0.197 \\
0.178 \\
0.487 \\
0.173\end{array}$ & $\begin{array}{l}1.522 \\
1.522 \\
1.522 \\
1.522\end{array}$ & $\begin{array}{l}40142 \\
40625 \\
39095 \\
41319\end{array}$ & $\begin{array}{l}2004 \\
1850 \\
4441 \\
1843\end{array}$ & $\begin{array}{l}1896 \\
1753 \\
3880 \\
1750\end{array}$ & 41799 & 630 & 630 & 40296 & 935 \\
\hline $\begin{array}{l}\text { AT-58-a } \\
\text { AT-58-b } \\
\text { AT-58-d }\end{array}$ & $\begin{array}{l}174.52 \\
174.52 \\
174.52\end{array}$ & $\begin{array}{l}0.763 \\
0.778 \\
0.780\end{array}$ & $\begin{array}{l}1.0 \mathrm{E}-03 \\
1.0 \mathrm{E}-03 \\
4.4 \mathrm{E}-03\end{array}$ & $\begin{array}{l}1.404 \\
1.417 \\
1.317\end{array}$ & $\begin{array}{l}9 \mathrm{E}-04 \\
8 \mathrm{E}-04 \\
2 \mathrm{E}-03\end{array}$ & $\begin{array}{c}8.15 \\
14.3 \\
6.66\end{array}$ & $\begin{array}{l}1 \mathrm{E}-02 \\
2 \mathrm{E}-02 \\
2 \mathrm{E}-02\end{array}$ & $\begin{array}{l}1.31 \\
1.31 \\
1.29\end{array}$ & $\begin{array}{l}0.376 \\
0.217 \\
0.463\end{array}$ & $\begin{array}{l}1.447 \\
1.447 \\
1.447\end{array}$ & $\begin{array}{l}65778 \\
74057 \\
71704\end{array}$ & $\begin{array}{r}12128 \\
6954 \\
19012\end{array}$ & $\begin{array}{r}9642 \\
6124 \\
13886\end{array}$ & 79857 & 4835 & 4351 & 70513 & 4926 \\
\hline $\begin{array}{l}\text { AT-62-a* } \\
\text { AT-62-b } \\
\text { AT-62-c } \\
\text { AT-62-d (det) }\end{array}$ & $\begin{array}{l}193.58 \\
193.58 \\
193.58 \\
193.58\end{array}$ & $\begin{array}{l}0.925 \\
0.837 \\
0.858 \\
0.857\end{array}$ & $\begin{array}{l}1.4 \mathrm{E}-03 \\
7.9 \mathrm{E}-04 \\
9.3 \mathrm{E}-04 \\
1.1 \mathrm{E}-03\end{array}$ & $\begin{array}{l}1.389 \\
1.371 \\
1.365 \\
1.367\end{array}$ & $\begin{array}{l}6 E-04 \\
6 E-04 \\
7 E-04 \\
6 E-04\end{array}$ & $\begin{array}{c}10.6 \\
7.42 \\
8.17 \\
8.41\end{array}$ & $\begin{array}{l}1 E-02 \\
6 E-03 \\
7 E-03 \\
1 E-02\end{array}$ & $\begin{array}{l}1.00 \\
1.48 \\
1.19 \\
1.22\end{array}$ & $\begin{array}{l}0.268 \\
0.509 \\
0.382 \\
0.381\end{array}$ & $\begin{array}{l}1.447 \\
1.447 \\
1.447 \\
1.447\end{array}$ & $\begin{array}{l}97006 \\
77598 \\
83736 \\
83889\end{array}$ & $\begin{array}{l}19537 \\
20020 \\
20178 \\
19222\end{array}$ & $\begin{array}{l}14923 \\
14594 \\
14885 \\
14352\end{array}$ & 163531 & 22406 & 18340 & 85557 & 8176 \\
\hline $\begin{array}{l}\text { AT-70-d } \\
\text { AT-70-e } \\
\text { AT-70-f (det) } \\
\text { AT-74-d } \\
\text { AT-70-g }\end{array}$ & $\begin{array}{l}220.03 \\
220.03 \\
220.03 \\
222.91 \\
220.03\end{array}$ & $\begin{array}{l}0.841 \\
0.850 \\
0.986 \\
0.846 \\
0.870\end{array}$ & $\begin{array}{l}8.7 \mathrm{E}-04 \\
1.1 \mathrm{E}-03 \\
1.9 \mathrm{E}-03 \\
9.0 \mathrm{E}-04 \\
8.5 \mathrm{E}-04\end{array}$ & $\begin{array}{l}1.312 \\
1.298 \\
1.132 \\
1.339 \\
1.302\end{array}$ & $\begin{array}{l}8 \mathrm{E}-04 \\
8 \mathrm{E}-04 \\
7 \mathrm{E}-04 \\
7 \mathrm{E}-04 \\
4 \mathrm{E}-04\end{array}$ & $\begin{array}{c}15.3 \\
11.1 \\
2.98 \\
15.1 \\
11.0\end{array}$ & $\begin{array}{l}1 \mathrm{E}-02 \\
1 \mathrm{E}-02 \\
4 \mathrm{E}-03 \\
1 \mathrm{E}-02 \\
9 \mathrm{E}-03\end{array}$ & $\begin{array}{l}3.09 \\
2.54 \\
4.32 \\
2.27 \\
2.29\end{array}$ & $\begin{array}{l}0.517 \\
0.595 \\
4.364 \\
0.389 \\
0.550\end{array}$ & $\begin{array}{l}1.529 \\
1.529 \\
1.529 \\
1.529 \\
1.529\end{array}$ & $\begin{array}{r}96740 \\
96681 \\
114798 \\
93970 \\
100146\end{array}$ & $\begin{array}{r}9878 \\
15215 \\
362555 \\
9709 \\
16669\end{array}$ & $\begin{array}{r}8523 \\
12208 \\
164606 \\
8402 \\
13204\end{array}$ & 95446 & 1465 & 1433 & 102205 & 6481 \\
\hline $\begin{array}{l}\text { AT-76-e } \\
\text { AT-76-f }\end{array}$ & $\begin{array}{l}241.07 \\
241.07\end{array}$ & $\begin{array}{l}0.993 \\
0.981\end{array}$ & $\begin{array}{l}1.2 \mathrm{E}-03 \\
1.2 \mathrm{E}-03\end{array}$ & $\begin{array}{l}1.328 \\
1.236\end{array}$ & $\begin{array}{l}8 \mathrm{E}-04 \\
7 \mathrm{E}-04\end{array}$ & $\begin{array}{l}9.11 \\
4.93\end{array}$ & $\begin{array}{l}9 \mathrm{E}-03 \\
5 \mathrm{E}-03\end{array}$ & $\begin{array}{l}1.26 \\
2.32\end{array}$ & $\begin{array}{l}0.420 \\
1.405\end{array}$ & $\begin{array}{l}1.447 \\
1.447\end{array}$ & $\begin{array}{l}118719 \\
116082\end{array}$ & $\begin{array}{r}39470 \\
182787\end{array}$ & $\begin{array}{l}25219 \\
48230\end{array}$ & 120515 & 731 & 732 & 117401 & 2637 \\
\hline $\begin{array}{l}\text { AT-79-e } \\
\text { AT-79-b } \\
\text { AT-79-c }\end{array}$ & $\begin{array}{l}283.17 \\
283.17 \\
283.17\end{array}$ & $\begin{array}{l}0.889 \\
0.750 \\
0.948\end{array}$ & $\begin{array}{l}1.0 \mathrm{E}-03 \\
9.2 \mathrm{E}-04 \\
2.2 \mathrm{E}-03\end{array}$ & $\begin{array}{l}1.205 \\
1.222 \\
1.083\end{array}$ & $\begin{array}{l}6 E-04 \\
5 E-04 \\
7 E-04\end{array}$ & $\begin{array}{c}11.7 \\
12.6 \\
3.74\end{array}$ & $\begin{array}{l}1 \mathrm{E}-02 \\
1 \mathrm{E}-02 \\
6 \mathrm{E}-03\end{array}$ & $\begin{array}{l}3.92 \\
4.31 \\
5.06\end{array}$ & $\begin{array}{l}0.907 \\
0.784 \\
3.917\end{array}$ & $\begin{array}{l}1.149 \\
1.149 \\
1.149\end{array}$ & $\begin{array}{r}118485 \\
85237 \\
109977\end{array}$ & $\begin{array}{r}32633 \\
13359 \\
334134\end{array}$ & $\begin{array}{r}22183 \\
10802 \\
163672\end{array}$ & 94347 & 14820 & 13345 & & \\
\hline $\begin{array}{l}\text { AT-92-c } \\
\text { AT-92-d* } \\
\text { AT-92-e } \\
\text { AT-92-f } \\
\text { AT-92-g }\end{array}$ & $\begin{array}{l}347.23 \\
347.23 \\
347.23 \\
347.23 \\
347.23\end{array}$ & $\begin{array}{l}1.054 \\
1.062 \\
1.050 \\
1.054 \\
1.056\end{array}$ & $\begin{array}{l}8.8 \mathrm{E}-04 \\
1.2 \mathrm{E}-03 \\
9.0 \mathrm{E}-04 \\
8.3 \mathrm{E}-04 \\
8.2 \mathrm{E}-04\end{array}$ & $\begin{array}{l}1.318 \\
1.321 \\
1.304 \\
1.310 \\
1.311\end{array}$ & $\begin{array}{l}6 \mathrm{E}-04 \\
9 \mathrm{E}-04 \\
6 \mathrm{E}-04 \\
6 \mathrm{E}-04 \\
8 \mathrm{E}-04\end{array}$ & $\begin{array}{l}33.9 \\
43.6 \\
25.8 \\
28.4 \\
30.9\end{array}$ & $\begin{array}{l}2 \mathrm{E}-02 \\
4 \mathrm{E}-02 \\
2 \mathrm{E}-02 \\
2 \mathrm{E}-02 \\
2 \mathrm{E}-02\end{array}$ & $\begin{array}{l}2.62 \\
2.55 \\
2.73 \\
2.61 \\
2.49\end{array}$ & $\begin{array}{l}0.249 \\
0.190 \\
0.339 \\
0.296 \\
0.260\end{array}$ & $\begin{array}{l}1.447 \\
1.447 \\
1.447 \\
1.447 \\
1.447\end{array}$ & $\begin{array}{l}152395 \\
155333 \\
153513 \\
153554 \\
154392\end{array}$ & $\begin{array}{r}11737 \\
9176 \\
16653 \\
14851 \\
13590\end{array}$ & $\begin{array}{r}10238 \\
8259 \\
13784 \\
12539 \\
11632\end{array}$ & 154866 & 2505 & 2438 & 153837 & 981 \\
\hline $\begin{array}{l}\text { AT-97-b } \\
\text { AT-97-c } \\
\text { AT-97-e } \\
\text { AT-97-f }\end{array}$ & $\begin{array}{l}395.00 \\
395.00 \\
395.00 \\
395.00\end{array}$ & $\begin{array}{l}1.261 \\
1.236 \\
1.177 \\
1.202\end{array}$ & $\begin{array}{l}4.1 \mathrm{E}-03 \\
1.4 \mathrm{E}-03 \\
1.3 \mathrm{E}-03 \\
1.7 \mathrm{E}-03\end{array}$ & $\begin{array}{l}1.302 \\
1.312 \\
1.263 \\
1.290\end{array}$ & $\begin{array}{l}6 E-04 \\
8 E-04 \\
7 E-04 \\
9 E-04\end{array}$ & $\begin{array}{c}11.3 \\
22.0 \\
9.93 \\
14.3\end{array}$ & $\begin{array}{l}3 \mathrm{E}-02 \\
2 \mathrm{E}-02 \\
9 \mathrm{E}-03 \\
2 \mathrm{E}-02\end{array}$ & $\begin{array}{l}1.59 \\
1.08 \\
1.19 \\
1.18\end{array}$ & $\begin{array}{l}0.543 \\
0.186 \\
0.430 \\
0.302\end{array}$ & $\begin{array}{l}1.496 \\
1.496 \\
1.496 \\
1.496\end{array}$ & $\begin{array}{l}243534 \\
228461 \\
214702 \\
218087\end{array}$ & $\begin{array}{r}378936 \\
60967 \\
221269 \\
101893\end{array}$ & $\begin{array}{l}78424 \\
37462 \\
66828 \\
48668\end{array}$ & 242587 & 37663 & 27432 & 226196 & 12957 \\
\hline
\end{tabular}


from hyperarid (reflected by halite deposition) to wetter conditions (reflected by the aad facies). We divide the $151 \mathrm{~m}$ of MIS5 into six stratigraphic intervals (I-1 to I-6) (Figs. 4, 5) reflecting the changing hydrology and climate in the watershed.

Interval I-1 (331-320 mblf, 137-133 ka) marks the transition from the relatively wet glacial MIS6 to the arid interglacial MIS5e (Termination 2 ; T2). The $\sim 11 \mathrm{~m}$ section comprises gypsum, clas-

Table 2

Age-depth model anchor ages.

\begin{tabular}{cr}
\hline $\begin{array}{l}\text { Depth } \\
\text { (mbif) }\end{array}$ & \multicolumn{1}{l}{$\begin{array}{l}\text { Age } \\
(\mathrm{ka})\end{array}$} \\
\hline 0 & $0.00 \pm 0.20$ \\
91 & $14.50 \pm 0.50$ \\
101 & $17.10 \pm 0.50$ \\
144.5 & $46.00 \pm 1.70$ \\
234.6 & $109.99 \pm 5.20$ \\
235 & $116.65 \pm 3.00$ \\
286.1 & $123.00 \pm 1.00$ \\
328 & $135.00 \pm 1.00$ \\
393 & $190.00 \pm 3.00$ \\
455 & $220.00 \pm 5.00$ \\
\hline
\end{tabular}

tic silts and aad. The $\delta^{18} \mathrm{O}$ of DSDDP core aragonites show shifts to lower values, marking the MIS6/5 transition from wet conditions (characterized mainly by aad and silt facies) to the arid interglacial conditions. T2 marks first major rise in sea level at the end of MIS6 (Grant et al., 2012) and overlaps with Heinrich Event 11 (Hemming, 2004), when the Northern Hemisphere ice sheet breakdown resulted in the discharge of iceberg armadas into the North Atlantic.

Interval I-2 (320-303 mblf, $\sim 133-128 \mathrm{ka})$ marks the initial phase of MIS5, when both high latitude and equatorial summer insolation rise towards their MIS5e peak. The $\sim 17 \mathrm{~m}$ section comprises interlayered halite and silty laminae. The aragonite $\delta^{18} \mathrm{O}$ values are similar to the Holocene (i.e., +2 to $+3 \% 0$ ), and both are lower than those of MIS6 (Fig. 4).

Interval I-3 (303-280 mblf, $\sim 128-122 \mathrm{ka}$ ) coincides with the peak NH summer insolation during MIS5e. This $\sim 23 \mathrm{~m}$ section is mainly comprised of silt layers (Fig. 1), with interspersed aragonite laminae. Despite its coincidence with the peak $\mathrm{NH}$ summer insolation and the highest sea level interval of MIS5e, the interval is nearly halite-free (Fig. 4), indicating a temporal dampening of hyperarid conditions and increased wetness in the watershed. It is coeval with anomalously low $\delta^{18} \mathrm{O}$ values in Soreq

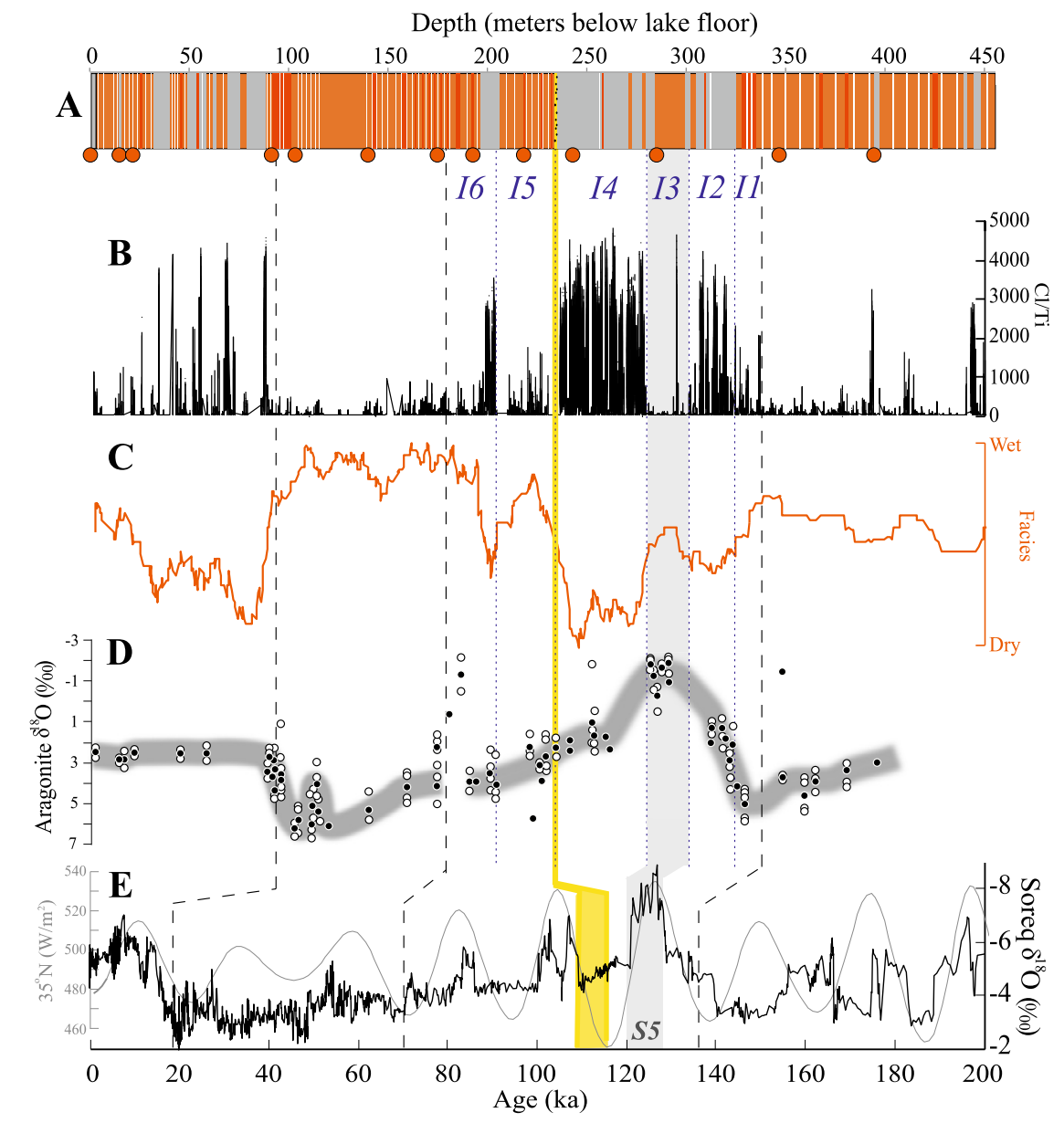

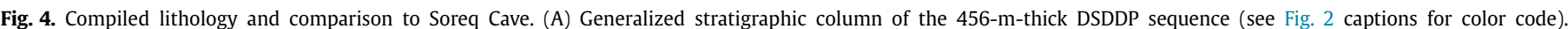

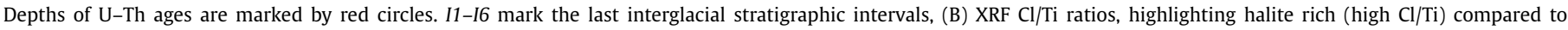

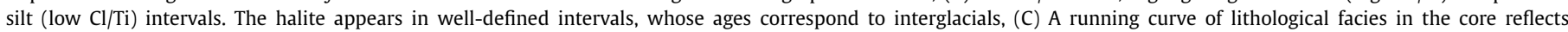

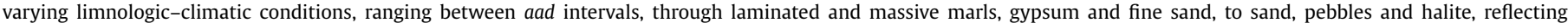

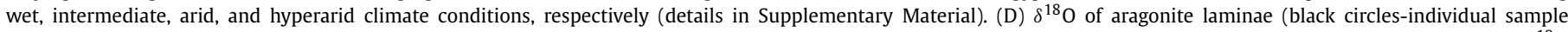

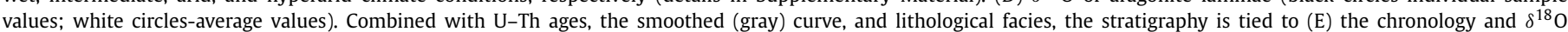

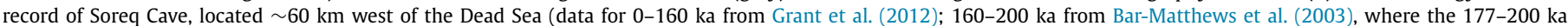

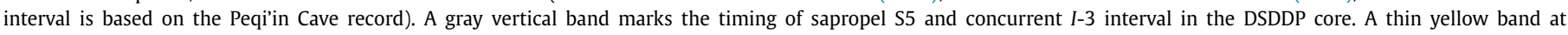

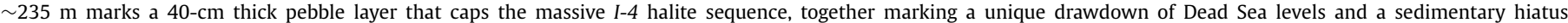
between $\sim 116-110 \mathrm{ka}$. (For interpretation of the references to color in this figure legend, the reader is referred to the web version of this article.) 


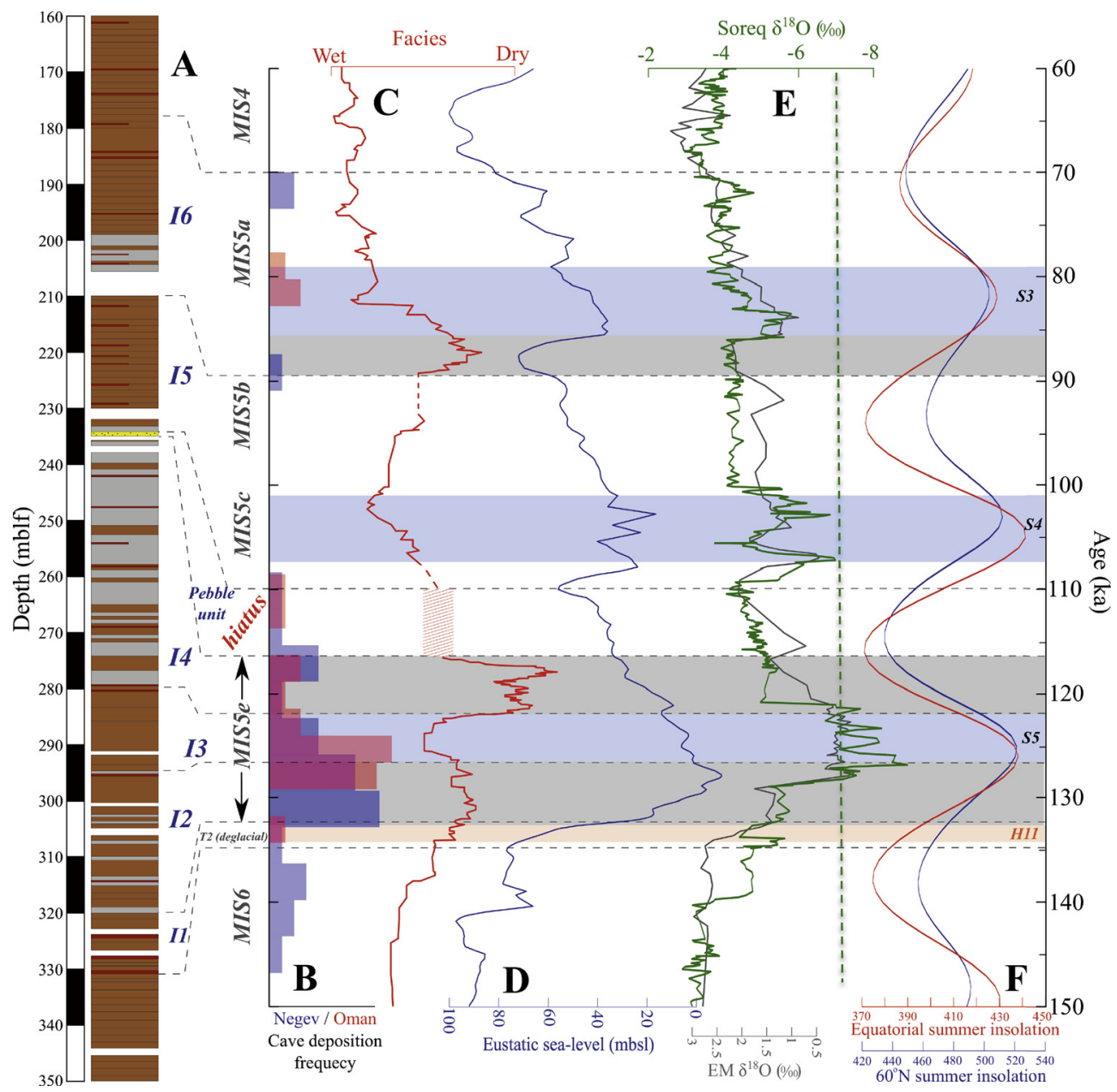

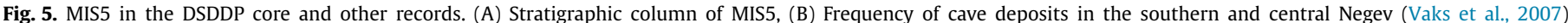

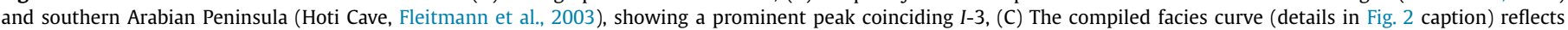

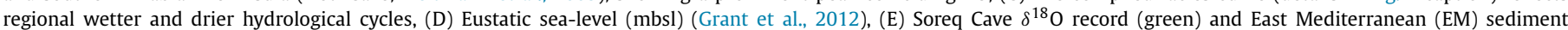

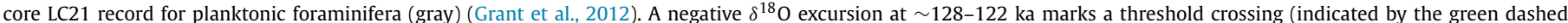

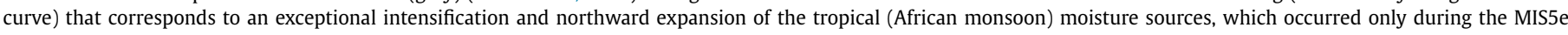

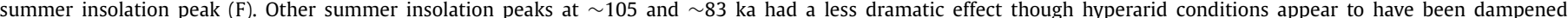

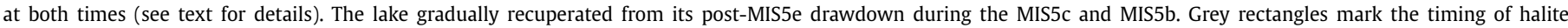

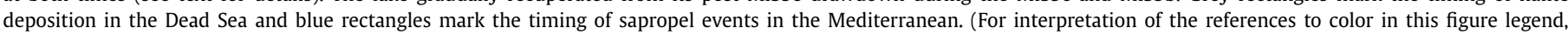
the reader is referred to the web version of this article.)

Cave speleothems (Fig. 4), associated with sapropel S5 in the Eastern Mediterranean (Bar-Matthews et al., 2003). The sapropel reflects extremely high Nile flow, caused by the unusually strong African monsoon associated with high equatorial summer insolation (Rohling et al., 2002; Rossignol-Strick, 1985). Like the Soreq speleothems, the aragonite laminae from the $I-3$ interval show extremely low $\delta^{18} \mathrm{O}$ values (between -2 and $0 \%$ ), lower than any other time interval in the Dead Sea record, including the Holocene (Fig. 4).

Interval I-4 ( 280-235 mblf, $\sim 122-116 \mathrm{ka})$ marks the most extreme arid spell known in the Dead Sea. This time interval coincides with the waning of the African monsoon during the latter part of MIS5e. Halite dominates over $\sim 45 \mathrm{~m}$ of section with thin interspersed silt and gypsum. This exceptionally long halitedominated interval is capped by a $\sim 40 \mathrm{~cm}$ thick pebble layer (at 235 mblf; Figs. 4, 5), whose appearance is similar to beach deposits along the modern Dead Sea shores (Fig. S2), suggesting its potential association with a paleo-shoreline. Together, the halite and pebble units represent a sequence of evaporation and lake level drop to below the paleo-elevation of the core site, in the deepest basin of the Dead Sea. In turn this suggests a major drawdown of the lake level at the end of MIS5e, which is supported by a $\sim 6-7$ kyr sedimentary hiatus in the DSDDP record (Fig. 3, Table 2).

Interval I-5 ( $\sim 234-210$ mblf, $\sim 110-93 \mathrm{ka})$, lies above the pebble unit and comprises a $\sim 20 \mathrm{~m}$ thick sequence of silt sediments and sporadic gypsum layers. This unit marks resumed wetness and gradual recovery of the lake level during MIS $5 c$ and $5 b$, coeval with sapropel event S4 in the Mediterranean, though the African monsoon, considered a potential driver of moisture delivery to the East Mediterranean-Levant region, was apparently weak during this time interval, as indicated by the gaps in several other regional records (Figs. 5, 7).

Interval I-6 ( $\sim 206-177$ mblf $\sim 90-70 \mathrm{ka})$ marks a return to hyperaridity during the transition into MIS5a, represented by halite and intermittent gypsum and silt. Subsequently, during the rest of MIS5a, a silt interval indicates more moderate conditions, possibly supported by a brief resumption intensified African monsoon activity (coinciding with S3 in the East Mediterranean; Rossignol-Strick, 1985), and the re-appearance of an aad-dominated sequence marks the onset of the last glacial cycle and the transition to the Lisan Formation. 


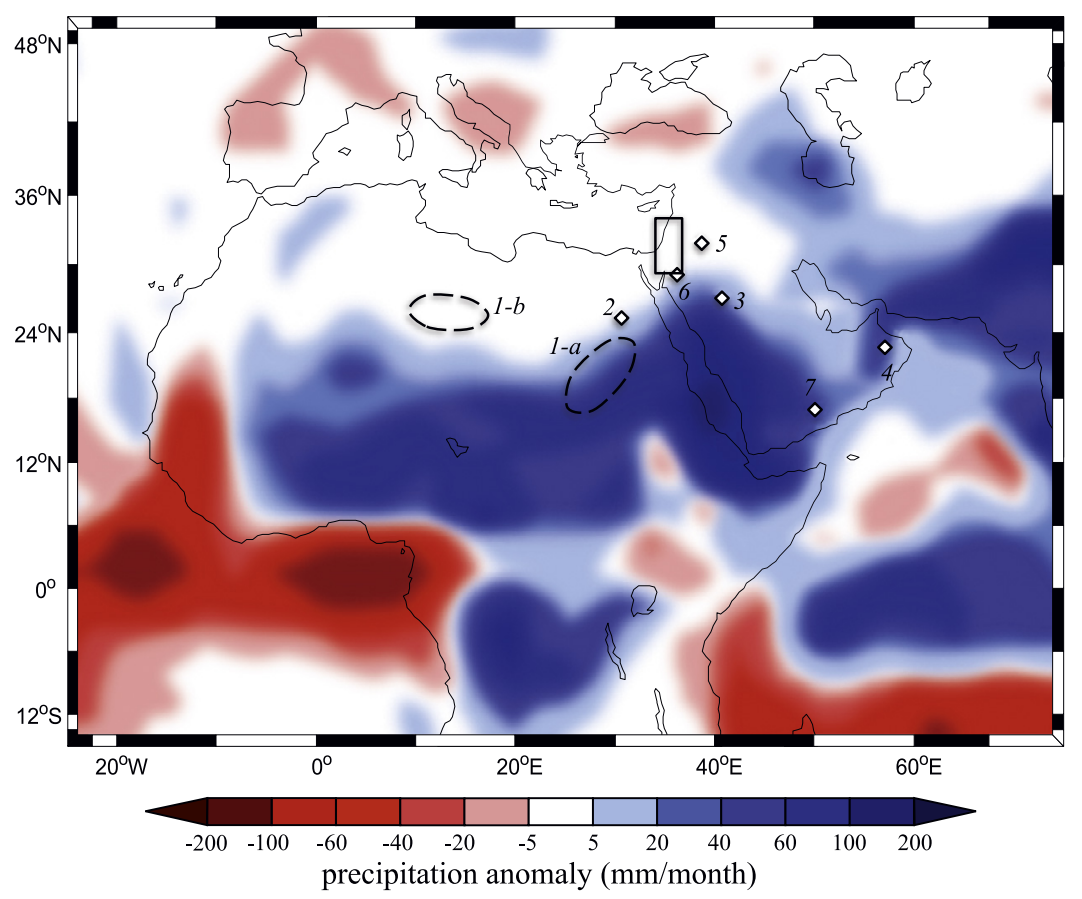

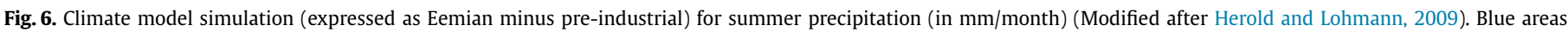

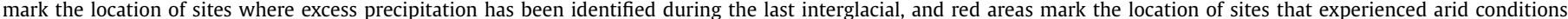

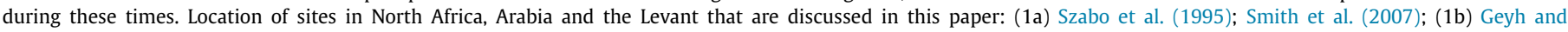

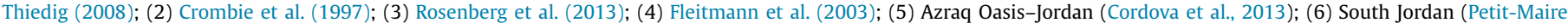

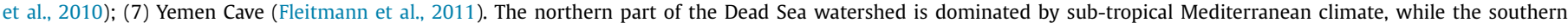

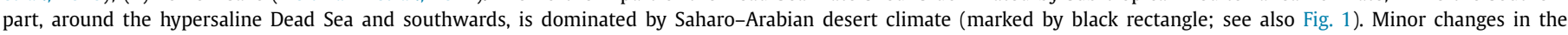

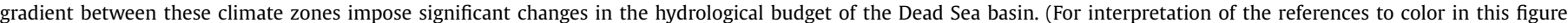
legend, the reader is referred to the web version of this article.)

\section{Climate control on the East Mediterranean-Levant hydrology}

The DSDDP sedimentary column provides a surprising perspective on the last interglacial climate in the Levant. Based on the long term relationships between sedimentary facies and climate in the Dead Sea basin, a more arid-related sediment facies (halite, gypsum and silt) would be expected to dominate during $\mathrm{NH}$ summer insolation maxima (MIS 5a, c, e) and wetter facies (silt and aad) during summer insolation minima (MIS5b, d). Accordingly, the MIS5e interglacial peak at $\sim 128-122 \mathrm{ka}$ is expected to reflect extreme hyperarid conditions. Yet, the DSDDP stratigraphy shows that the MIS5e insolation peak is coincident with relative wetness in the Levant, which is also coeval with the S5 sapropel in the Mediterranean, it self associated with an intensification of the African monsoon.

The identification of the source of the MIS5e moisture to the Dead Sea watershed poses a perplexing question. The Mediterranean sapropel layers are considered to be the result of enhanced Nile River flow, fed by the remote precipitation increase in low latitudes, associated with an intense African summer monsoon (Rohling et al., 2002; Rossignol-Strick, 1985). Yet, the ability of monsoon-related southern-derived rains to directly impact the Dead Sea watershed is not trivial, considering the relatively high latitude of the lake and the distance from tropical Africa. However, a significant northern excursion of summer monsoon rainfall is displayed in recent general circulation models (e.g., Herold and Lohmann, 2009), suggesting that the African monsoon influence could have reached as far north as the northern Arabian Peninsula and the southern end of the Dead Sea watershed, triggering summer rains of up to $\sim 30-60 \mathrm{~mm}$ per summer (Fig. 6). It should also be noted that in recent years, the most significant floods in the southern Negev-Arava are the result of rainstorms associated with "tropical moisture plumes" crossing north Africa and impacting the deserts of the Middle East (Kahana et al., 2002; Rubin et al., 2007; Ziv, 2001). These storms can therefore provide an indication of an alternative, or complementary, efficient mechanism for the impact of southern sources of humidity on the Dead Sea watershed. Because of their localized impact however, they are not necessarily associated with a regional scale northsouth geographic trend, as indicated from their modern input in Sinai, Negev, Jordan, and NW Arabia deserts (Rubin et al., 2007). Our hypothesis that southern rainfall sources affected the Dead Sea watershed during the MIS5e peak is corroborated by other regional hydroclimate archives (Figs. 1, 6, 7) such as southern Negev Desert speleothems (Vaks et al., 2010, 2007), travertine-spring deposits in the Arava valley (Livnat and Kronfeld, 1985; Waldmann et al., 2010), and MIS5e ages of calcification processes in the Red Sea coral reefs indicating enhanced coastal fresh groundwater activity in the currently hyperarid Gulf of Aqaba (Lazar and Stein, 2011). The evidence from the Negev-Arava-Red Sea archives are supported by additional evidence from cave deposits in the southern Arabian Peninsula (Fleitmann et al., 2011, 2003), and spring and possibly lacustrine or groundwater overflow deposits in Northern and Eastern Sahara (Geyh and Thiedig, 2008; McKenzie, 1993; Szabo et al., 1995), Arabia (Rosenberg et al., 2013), and south Jordan (Petit-Maire et al., 2010; note however, a revised evaluation of the latter by Catlett, 2014). Collectively, these observations indicate that tropical-derived precipitation could have reached as far north as the Dead Sea watershed during MIS5e, inverting the common hyperaridy persisting during full interglacials. As a result, the Dead Sea system was maintained above the annual hydrologic threshold of halite precipitation through the MIS5e peak, as reflected by core interval $I-3$. The collapse of the hydrological system was therefore postponed for several thousand years, until the effect of the southern humidity sources weakened (Fig. 5b), and the annual inflow to the lake fell below the threshold of halite precipitation. 


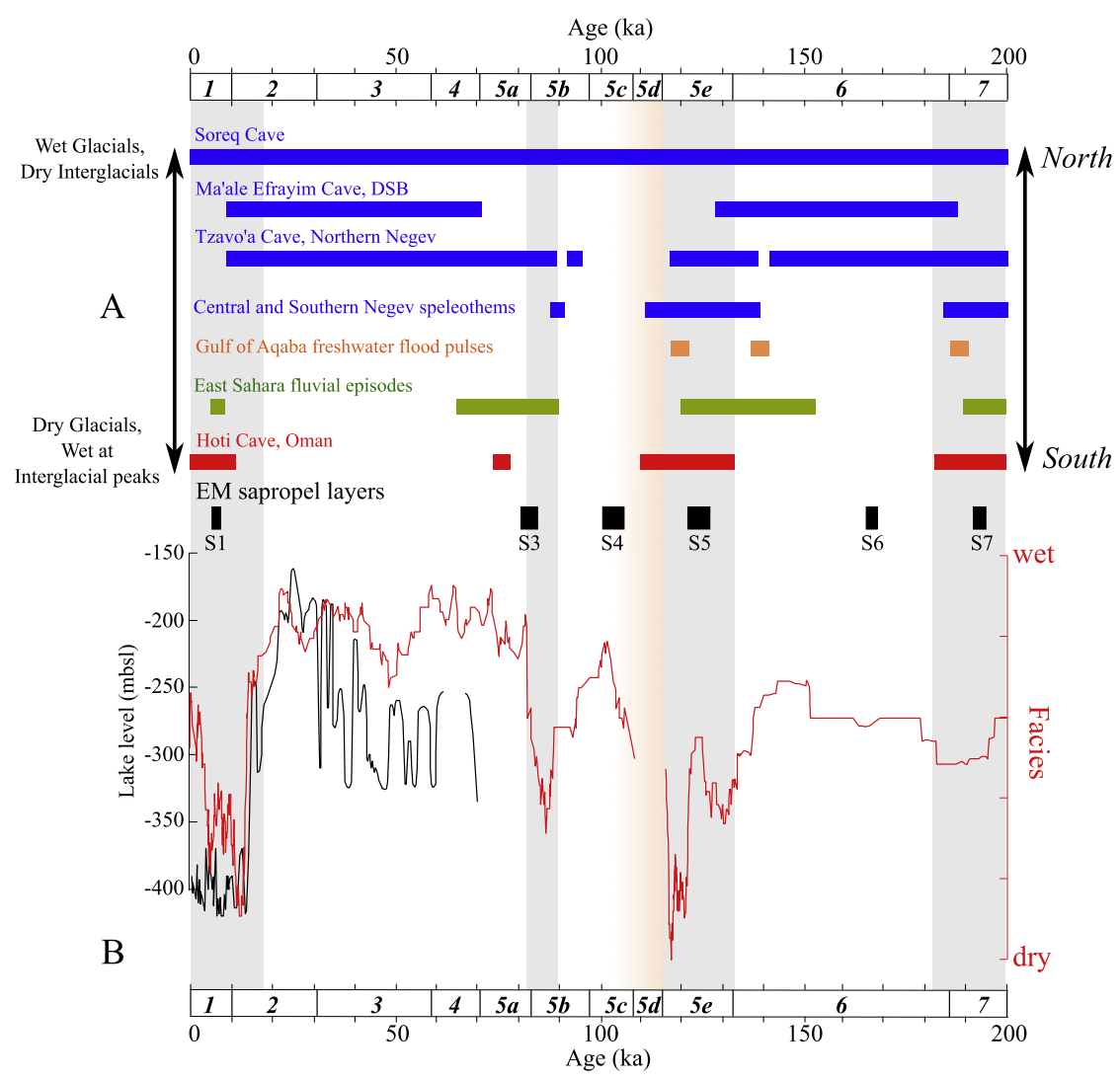

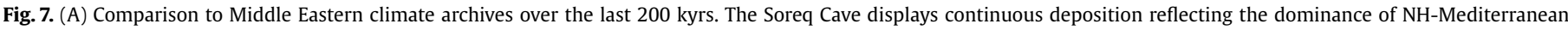

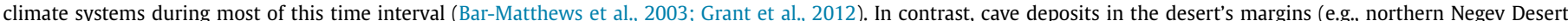

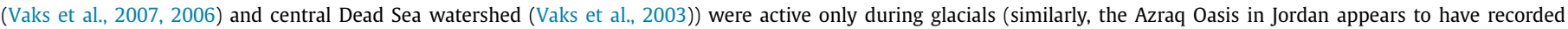

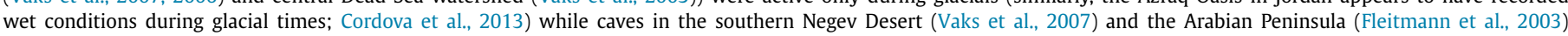

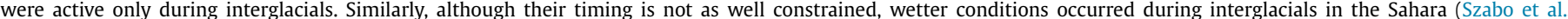

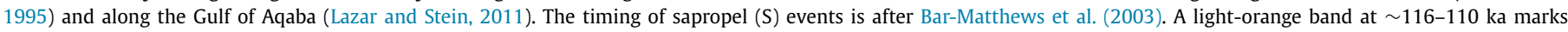

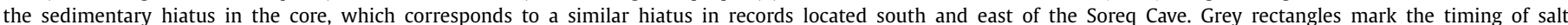

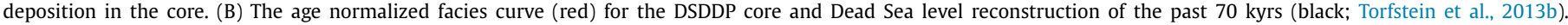

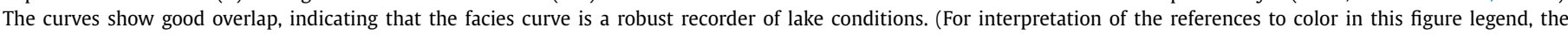
reader is referred to the web version of this article.)

An effective incursion of tropic-derived precipitation systems as far north as the southern Dead Sea watershed must have been limited to the peak of MIS5e ( $\sim 128-122 \mathrm{ka}$, coeval with sapropel S5 and DSDDP Interval I-3). Indeed, the S5-associated wet anomaly is well documented in both the DSDDP core and Soreq Cave, where the lightest $\delta^{18} \mathrm{O}$ values are recorded in both archives (Figs. 4, 5 ). Because the main source of precipitation in the Levant is the Eastern Mediterranean, both records show a remarkably similar variability in $\delta^{18} \mathrm{O}$ values, following the pattern of East Mediterranean planktonic foraminifera $\delta^{18} \mathrm{O}$ (Kolodny et al., 2005). During the peak of MIS5e, the enhanced freshwater inflow, which was delivered to the Mediterranean primarily by the Nile River and to a lesser extent to the southern Levant by southern rain, imposed uniquely low $\delta^{18} \mathrm{O}$ values that stem from the input of far traveled precipitation.

The African monsoon contribution to Arabia declined gradually from the MIS5e peak until $\sim 110 \mathrm{ka}$, when it became negligible, as expressed by the declining frequency of cave deposits in Oman (Fleitmann et al., 2003) and in the Negev Desert (Vaks et al., 2007, 2006) (Fig. 5b). This resulted in a significant lake drop and a major phase of salt deposition, as reflected in the I-4 core interval and the sedimentary hiatus that follows it, between $\sim 116-110 \mathrm{ka}$. This lake-level drop marks one of them most extreme regional aridity cycles associated with the combined effect of a NH-dominated hyperarid interglacial, a weakening African monsoon, and the already moisture-deprived hydrological system of the Dead Sea watershed.
Recovery of the Dead Sea watershed from hyperarid conditions might be expected during MIS5d ( $116-110 \mathrm{ka})$, which is considered less extreme than MIS5e. Yet the hydrological system was still water-deprived and lake levels remained extremely low. Following the shift into MIS5c ca. $110 \mathrm{ka}$, the DSDDP record displays renewed deposition of silt and gypsum beds, indicating a limited and gradual recovery of the hydrological system. It should be noted that spring and cave deposits in the SaharoArabian area generally ceased forming during this time interval (Fig. 7), reflecting continued hyperarid conditions. Thereafter, the gradual recovery continued through MIS5b, when global and regional climate conditions became more moderate, but terminated abruptly with the deposition of massive halite ca. $\sim 90-85 \mathrm{ka}$ (206-199 mblf). This arid spell was followed by relatively moderate conditions that could be related to the combination of a resumed, albeit brief, contribution of the southern rains during the peak of MIS5a ( $85-80 \mathrm{ka})$, coinciding with sapropel S3 in the Mediterranean (Grant et al., 2012; Rossignol-Strick, 1985), and the gradual NH shift toward the MIS4 glacial stage, which imposed much wetter conditions in the Dead Sea watershed.

\section{Summary and conclusions}

Recent drilling by the Dead Sea Deep Drilling Project in the deepest basin of the hypersaline Dead Sea at $\sim 723 \mathrm{mbsl}$ recovered 
sediment cores that record the late Quaternary limnological history of the lake and the hydro-climatic history of its watershed between the present day and MIS7 at $200 \mathrm{ka}$. This study focuses on the last interglacial period (MIS5), including new observations regarding the impacts of particularly intense precession-driven monsoons at the mid subtropical latitude of the Dead Sea watershed.

Relative wetness is reflected by sequences of alternating aragonite and silty laminae (aad facies), and increasing aridity is reflected by decreasing amounts of aragonite and a corresponding increase in the content of laminated detritus (ld facies), then gypsum, then halite. The chronology of the DSDDP core has been established by integration of U-Th ages of primary aragonite, $\delta^{18} \mathrm{O}$ oxygen isotope stratigraphy and comparison of the main lithological units to regional and global archives. The chronostratigraphy shows that the aad facies is largely associated with glacials, while halite deposition marks regional aridity and lake drops uniquely associated with interglacials.

The sediment sequence deposited in the Dead Sea during MIS5 records the interplay between the effects of $\mathrm{NH}$ climate and intense precession driven African moisture sources on the climate of the Eastern Mediterranean-Levant corridor. The NH climatology imposes arid interglacials and wetter glacials in the region, while particularly intense African monsoons, such as the one that generated sapropel S5 in the Eastern Mediterranean during the peak of MIS5e, moderated the aridity otherwise expected in the Dead Sea basin. The new findings confirm that in the absence of these tropical effects, the Levant corridor would have been extremely hyperarid during MIS5e, leading to an early catastrophic drop of the Dead Sea. Instead, a major lake drawdown took place during the late stages of MIS5e.

In the context of human prehistory, wetness in the region coeval with an intensified African monsoon may have facilitated hominid migration out of Africa (e.g., Frumkin et al., 2011; Lazar and Stein, 2011; Vaks et al., 2007; Waldmann et al., 2010; Walter et al., 2000), while otherwise a regional climate barrier of aridity persisted across the Levant, Arabia and Eastern Sahara during much of MIS5. Finally, the findings reported here support general circulation model results (e.g., Held and Soden, 2006) predicting that warmer mean global temperatures may exacerbate an important resource problem by causing a decline in Levant fresh water availability.

\section{Acknowledgements}

We acknowledge the International Continental Scientific Drilling Foundation (ICDP), Israel Science Foundation (ISF), and US NSF (grant EAR 11-15312 to SLG and Emi Ito) for funding the coring, and DOSECC (Drilling, Observation and Sampling of the Earth's Continental Crust) for performing the drilling operation. The cores were opened and described by a large team under the auspices of the Professor A. Brauer at GFZ-Potsdam. We wish to thank the many participants who took part in the drilling operations, core opening and sampling. Rachel Lupien carefully separated and prepared aragonite laminae for oxygen isotope analyses. The scientific study is supported by the Comer Science \& Education Foundation (grant \#CP69 to AT), the USA-Israel Bi-National Science Foundation (BSF, grant \#2010375 to SLG and MS), the Dead Sea Drill Excellence Center of the Israel Science Foundation (grant \# 1736/11 to YE, Boaz Lazar, and Yigal Erel), the National Oceanic and Atmospheric Administration (grant NA10OAR4320137 to YK), and the Storke Endowment at Columbia University (to SLG). This is Dead Sea Excellence Center contribution \#4 and LDEO contribution \#7849. Two anonymous reviewers are thanked for providing helpful and constructive comments.

\section{Appendix A. Supplementary material}

Supplementary material related to this article can be found online at http://dx.doi.org/10.1016/j.epsl.2014.12.013.

\section{References}

Bar-Matthews, M., 2014. History of water in the Middle East and North Africa. In: Archaeology and Anthropology. Elsevier Ltd., pp. 109-128.

Bar-Matthews, M., Ayalon, A., Gilmour, M., Matthews, A., Hawksworth, C.J., 2003. Sea-land oxygen isotopic relationships from planktonic foraminifera and speleothems in the Eastern Mediterranean region and their implication for paleorainfall during. Geochim. Cosmochim. Acta 67, 3181-3199.

Bartov, Y., Stein, M., Enzel, Y., Agnon, A., Reches, Z., 2002. Lake levels and sequence stratigraphy of lake Lisan, the late Pleistocene precursor of the Dead Sea. Quat. Res. 57, 9-21.

Bartov, Y., Goldstein, S.L., Stein, M., Enzel, Y., 2003. Catastrophic arid episodes in the Eastern Mediterranean linked with the North Atlantic Heinrich events. Geology 31, 439-442.

Begin, Z., Ehrlich, A., Nathan, Y., 1974. Lake Lisan, the Pleistocene precursor of the Dead Sea. Geol. Surv. Isr. Bull. 63, 1-30.

Bookman (Ken-Tor), R., Enzel, Y., Agnon, A., Stein, M., 2004. Late Holocene lake levels of the Dead Sea. Geol. Soc. Am. Bull. 116, 555.

Catlett, G.A., 2014. Pluvial deposits in Mudawwara, Jordan and their implications for Mediterranean and monsoonal precipitation in the Levant. MSc thesis. Miami University

Cordova, C.E., Nowell, A., Bisson, M., Ames, C.J.H., Pokines, J., Chang, M., al-Nahar, M., 2013. Interglacial and glacial desert refugia and the Middle Paleolithic of the Azraq Oasis, Jordan. Quat. Int. 300, 94-110.

Crombie, M.K., Arvidson, R.E., Sturchio, N.C., El Alfy, Z., Zeid, K.A., 1997. Age and isotopic constraints on Pleistocene pluvial episodes in the Western Desert, Egypt. Palaeogeogr. Palaeoclimatol. Palaeoecol. 130, 337-355

Enzel, Y., Amit, R., Dayan, U., Crouvi, O., Kahana, R., Ziv, B., Sharon, D., 2008. The climatic and physiographic controls of the eastern Mediterranean over the late Pleistocene climates in the southern Levant and its neighboring deserts. Glob. Planet. Change 60, 165-192.

Fleitmann, D., Burns, S.J., Neff, U., Mangini, A., Matter, A., 2003. Changing moisture sources over the last 330,000 years in Northern Oman from fluid-inclusion evidence in speleothems. Quat. Res. 60, 223-232.

Fleitmann, D., Burns, S.J., Pekala, M., Mangini, A., Al-Subbary, A., Al-Aowah, M., Kramers, J., Matter, A., 2011. Holocene and Pleistocene pluvial periods in Yemen, southern Arabia. Quat. Sci. Rev. 30, 783-787.

Frumkin, A., Bar-Yosef, O., Schwarcz, H.P., 2011. Possible paleohydrologic and paleoclimatic effects on hominin migration and occupation of the Levantine Middle Paleolithic. J. Hum. Evol. 60, 437-451.

Geyh, M.A., Thiedig, F., 2008. The Middle Pleistocene Al Mahrúqah Formation in the Murzuq Basin, northern Sahara, Libya evidence for orbitally-forced humid episodes during the last 500,000 years. Palaeogeogr. Palaeoclimatol. Palaeoecol. 257, 1-21.

Grant, K.M., Rohling, E.J., Bar-Matthews, M., Ayalon, A., Medina-Elizalde, M., Bronk Ramsey, C., Satow, C., Roberts, A.P., 2012. Rapid coupling between ice volume and polar temperature over the past 150,000 years. Nature 491, 744-747.

Haase-Schramm, A., Goldstein, S.L., Stein, M., 2004. U-Th dating of Lake Lisan (late Pleistocene dead sea) aragonite and implications for glacial east Mediterranean climate change. Geochim. Cosmochim. Acta 68, 985-1005.

Haliva-Cohen, A., Stein, M., Goldstein, S.L., Sandler, A., Starinsky, A., 2012. Sources and transport routes of fine detritus material to the Late Quaternary Dead Sea basin. Quat. Sci. Rev. 50, 55-70.

Held, I., Soden, B., 2006. Robust responses of the hydrological cycle to global warming. J. Climate 19, 5686-5699.

Hemming, S., 2004. Heinrich events: massive late Pleistocene detritus layers of the North Atlantic and their global imprint. Rev. Geophys. 42, 2003RG000128.

Herold, M., Lohmann, G., 2009. Eemian tropical and subtropical African moisture transport: an isotope modelling study. Clim. Dyn. 33, 1075-1088.

Kahana, R., Ziv, B., Enzel, Y., Dayan, U., 2002. Synoptic climatology of major floods in the Negev Desert, Israel. Int. J. Climatol. 22, 867-882.

Kaufman, A., 1971. U-Series dating of Dead Sea Basin carbonates. Geochim. Cosmochim. Acta 35, 1269-1281.

Kaufman, A., Yechieli, Y., Gardosh, M., 1992. Reevaluation of the lake-sediment chronology in the Dead Sea basin, Israel, based on new ${ }^{230} \mathrm{Th} / \mathrm{U}$ dates. Quat. Res. 304, 292-304

Kolodny, Y., Stein, M., Machlus, M., 2005. Sea-rain-lake relation in the Last Glacial East Mediterranean revealed by $\delta^{18} \mathrm{O}-\delta^{13} \mathrm{C}$ in Lake Lisan aragonites. Geochim. Cosmochim. Acta 69, 4045-4060.

Kushnir, Y., Stein, M., 2010. North Atlantic influence on 19th-20th century rainfall in the Dead Sea watershed, teleconnections with the Sahel, and implication for Holocene climate fluctuations. Quat. Sci. Rev. 29, 3843-3860.

Lazar, B., Stein, M., 2011. Freshwater on the route of hominids out of Africa revealed by U-Th in Red Sea corals. Geology 39, 1067-1070. 
Lisiecki, L.E., Raymo, M.E., 2005. A Pliocene-Pleistocene stack of 57 globally distributed benthic $\delta^{18} \mathrm{O}$ records. Paleoceanography 20, PA1003.

Livnat, A., Kronfeld, J., 1985. Paleoclimatic implications of U-series dates for lake sediments and travertines in the Arava Rift Valley, Israel. Quat. Res. 24, 164-172.

McKenzie, J.a., 1993. Pluvial conditions in the eastern Sahara following the penultimate deglaciation: implications for changes in atmospheric circulation patterns with global warming. Palaeogeogr. Palaeoclimatol. Palaeoecol. 103, 95-105.

Migowski, C., Stein, M., Prasad, S., Negendank, J.F.W., Agnon, A., 2006. Holocene climate variability and cultural evolution in the Near East from the Dead Sea sedimentary record. Quat. Res. 66, 421-431.

Neev, D., Emery, K.O., 1967. The Dead Sea, depositional processes and environments of evaporites. Geol. Surv. Isr. Bull. 41, 147 pp.

Neugebauer, I., Brauer, A., Schwab, M.J., Waldmann, N., Enzel, Y., Kitagawa, H., Torfstein, A., Frank, U., Dulski, P., Agnon, A., Ariztegui, D., Ben-Avraham, Z., Goldstein, S.L., Stein, M., 2014. Lithology of the long sediment record recovered by the ICDP Dead Sea Deep Drilling Project (DSDDP). Quat. Sci. Rev. 102, 149-165.

Petit-Maire, N., Carbonel, P., Reyss, J.L., Sanlaville, P., Abed, a., Bourrouilh, R., Fontugne, M., Yasin, S., 2010. A vast Eemian palaeolake in Southern Jordan $\left(29^{\circ} \mathrm{N}\right)$. Glob. Planet. Change 72, 368-373.

Prasad, S., Vos, H., Negendank, J.F.W., Waldmann, N., Goldstein, S.L., Stein, M., 2004. Evidence from Lake Lisan of solar influence on decadal- to centennial-scale climate variability during marine oxygen isotope stage 2. Geology 32, 581.

Rohling, E.J., Cane, T.R., Cooke, S., Sprovieri, M., Bouloubassi, I., Emeis, K.C., Schiebel, R., Kroon, D., Jorissen, F.J., Lorre, a., Kemp a, E.S., 2002. African monsoon variability during the previous interglacial maximum. Earth Planet. Sci. Lett. 202, 61-75.

Rosenberg, T.M., Preusser, F., Risberg, J., Plikk, A., Kadi, K.A., Matter, A., Fleitmann, D., 2013. Middle and Late Pleistocene humid periods recorded in palaeolake deposits of the Nafud desert, Saudi Arabia. Quat. Sci. Rev. 70, 109-123.

Rossignol-Strick, M., 1985. Mediterranean Quaternary sapropels, an immediate response of the African monsoon to variation of insolation. Palaeogeogr. Palaeoclimatol. Palaeoecol. 49, 237-263.

Rubin, S., Ziv, B., Paldor, N., 2007. Tropical plumes over Eastern North Africa as a Source of rain in the Middle East. Mon. Weather Rev. 135, 4135-4148.

Schramm, A., Stein, M., Goldstein, S., 2000. Calibration of the $14{ }^{\circ} \mathrm{C}$ time scale to $>40$ ka by ${ }^{234} \mathrm{U}-{ }^{230} \mathrm{Th}$ dating of Lake Lisan sediments (last glacial Dead Sea). Earth Planet. Sci. Lett. 175, 27-40.

Smith, J.R., Hawkins, A.L., Asmerom, Y., Polyak, V., Giegengack, R., 2007. New age constraints on the Middle Stone Age occupations of Kharga Oasis, Western Desert, Egypt. J. Hum. Evol. 52, 690-701.

Stein, M., 2001. The sedimentary and geochemical record of Neogene-Quaternary water bodies in the Dead Sea Basin - inferences for the regional paleoclimatic history. J. Paleolimnol. 26, 271-282.

Stein, M., Starinsky, A., Katz, A., Goldstein, S.L., Machlus, M., Schramm, A., 1997. Strontium isotopic, chemical, and sedimentological evidence for the evolution of Lake Lisan and the Dead Sea. Geochim. Cosmochim. Acta 61, 3875-3992.

Stein, M., Torfstein, A., Gavrieli, I., Yechieli, Y., 2010. Abrupt aridities and salt deposition in the post-glacial Dead Sea and their North Atlantic connection. Quat. Sci. Rev. 29, 567-575.
Szabo, B., Haynes, C., Maxwell, T., 1995. Ages of Quaternary pluvial episodes determined by uranium-series and radiocarbon dating of lacustrine deposits of Eastern Sahara. Palaeogeogr. Palaeoclimatol. Palaeoecol. 113, 227-242.

Torfstein, A., Gavrieli, I., Stein, M., 2005. The sources and evolution of sulfur in the hypersaline Lake Lisan (paleo-Dead Sea). Earth Planet. Sci. Lett. 236, 61-77.

Torfstein, A., Gavrieli, I., Katz, A., Kolodny, Y., Stein, M., 2008. Gypsum as a monitor of the paleo-limnological-hydrological conditions in Lake Lisan and the Dead Sea. Geochim. Cosmochim. Acta 72, 2491-2509.

Torfstein, A., Haase-Schramm, A., Waldmann, N., Kolodny, Y., Stein, M., 2009. Useries and oxygen isotope chronology of the mid-Pleistocene Lake Amora (Dead Sea basin). Geochim. Cosmochim. Acta 73, 2603-2630.

Torfstein, A., Goldstein, S., Kagan, E.J., Stein, M., 2013a. Integrated multi-site UTh chronology of the last glacial Lake Lisan. Geochim. Cosmochim. Acta 104, 210-231.

Torfstein, A., Goldstein, S., Stein, M., Enzel, Y., 2013b. Impacts of abrupt climate changes in the Levant from Last Glacial Dead Sea levels. Quat. Sci. Rev. 69, 1-7.

Vaks, A., Bar-Matthews, M., Ayalon, A., Schilman, B., Gilmour, M., Hawkesworth, C.J. Frumkin, A., Kaufman, A., Matthews, A., 2003. Paleoclimate reconstruction based on the timing of speleothem growth and oxygen and carbon isotope composition in a cave located in the rain shadow in Israel. Quat. Res. 59, 182-193.

Vaks, A., Bar-Matthews, M., Ayalon, A., Matthews, A., Frumkin, A., Dayan, U., Halicz, L., Almogi-Labin, A., Schilman, B., 2006. Paleoclimate and location of the border between Mediterranean climate region and the Saharo-Arabian Desert as revealed by speleothems from the northern Negev Desert, Israel. Earth Planet. Sci. Lett. 249, 384-399.

Vaks, A., Bar-Matthews, M., Ayalon, A., Matthews, A., Halicz, L., Frumkin, A., 2007 Desert speleothems reveal climatic window for African exodus of early modern humans. Geology 35, 831.

Vaks, A., Bar-Matthews, M., Matthews, A., Ayalon, A., Frumkin, A., 2010. Middle-Late Quaternary paleoclimate of northern margins of the Saharan-Arabian Desert: reconstruction from speleothems of Negev Desert, Israel. Quat. Sci. Rev. 29, 2647-2662.

Waldmann, N., Stein, M., Ariztegui, D., Starinsky, A., 2009. Stratigraphy, depositional environments and level reconstruction of the last interglacial Lake Samra in the Dead Sea basin. Quat. Res. 72, 1-15.

Waldmann, N., Torfstein, A., Stein, M., 2010. Northward intrusions of low- and midlatitude storms across the Saharo-Arabian belt during past interglacials. Geology 38, 567-570.

Walter, R., Buffler, R., Bruggemann, J., Guillaume, M., Berhe, S., Negassi, B., Libsekal, Y., Cheng, H., Edwards, R., von Cosel, R., Neraudeau, D., Gagnon, M., 2000. Early human occupation of the Red Sea coast of Eritrea during the last interglacial. Nature 405, 65-69.

Yechieli, Y., Magaritz, M., Levy, Y., Weber, U., Kafri, U., Woelfli, W., Bonani, G., 1993. Late Quaternary geological history of the Dead Sea area, Israel. Quat. Res. 39 59-67.

Ziv, B., 2001. A subtropical rainstorm associated with a tropical plume over Africa and the Middle-East. Theor. Appl. Climatol. 102, 91-102.

Ziv, B., Dayan, U., Kushnir, Y., Roth, C., Enzel, Y., 2006. Regional and global atmospheric patterns governing rainfall in the southern Levant. Int. J. Climatol. 26, 55-73. 